\title{
THE DESIGN OF LOW FREQUENCY HEAVY ION RFQ RESONATORS *
}

\author{
A. Schempp, H. Vormann, U. Beisel, H. Deitinghoff, O. Engels, D. Li ${ }^{+}$ \\ Johann Wolfgang Goethe Universität, \\ Frankfurt am Main, Germany \\ ${ }^{+}$on leave from Department of Technical Physics, Peking University, Beijing, China
}

\section{Abstract}

The development of the 4-Rod-RFQ as injector for low charged heavy ions has demonstrated that structures with spiral shaped and straight stems can be made highly efficient, very stable and compact. The design of the RFQ structures and results of impedance optimizations and the effects of monopole and dipole mode generation will be discussed.

\section{INTRODUCTION}

Because of improvements in ion source technology a new layout of the GSI High Current Injector can use higher charged ions: $\mathrm{U}^{4+}$ instead of $\mathrm{U}^{2+}$, as used for the "old" High Current Injector design. The old HCI was planned as a 27 MHz-RFQ for $\mathrm{U}^{2+}$ as injector for the Wideroe and a stripper for producing $\mathrm{U}^{10+}$ with a necessary current of $25 \mathrm{~mA}$. In the new concept a beam current of only $10 \mathrm{~mA} \mathrm{U}^{4+}$ is required, because a stripper is not needed any more, the beam will be injected directly into a new IH-Linac.

\section{RF-CALCULATIONS}

The specific parameters of the rf-properties of the different RFQ resonators have been checked by model measurements and calculations with the program MAFIA. Special considerations has been given to dipole effects, distribution of losses along the structure, and the influence of electrode-capacitance on the rf-parameters.

\section{A. Capacitance of electrodes}

The capacitance of the electrodes has a strong influence on the resonance frequency of the resonator. A resonator loaded with a big capacitance usually is less efficient, the Rpvalue (shuntimpedance) is smaller. Although the vane-shaped geometry has $20 \%$ more capacitance than the circular cylindric one, the impedance is nearly equal, the greater surface produces less losses. Table 1 shows a comparison of resonators with vane- respectively rod-shaped electrodes. The numeric calculations of characteristic resonator-data was done with the programs MAFIA and OPERA. It is a question of manufacture and cooling, which shape of electrodes will be chosen. So mechanical parameters and costs may determine the choice.

* supported by the BMFT

\begin{tabular}{|c|c|c|}
\hline & Vanes & Rods \\
\hline Frequency / MHz & 30.50 & 32.24 \\
\hline Quality-Factor & 12700 & 11500 \\
\hline Shunt-imp./k $\Omega \mathrm{m}$ & 277.3 & 258.5 \\
\hline
\end{tabular}

Table 1: RF-Parameters for different electrode-shapes.

\section{B. Dipole effects}

Dipole contributions in the electrode fields correspond to differences in the potential of the electrodes, e.g. the beam axis is not on zero potential. They can be a reason for beam losses, depending on their strength and beam emittances. Dipole-components of the electrode-voltage can be reduced by shaping the stems with an angle $\alpha$ as shown in fig. 1, which has been used in all operating four-rod RFQs [1]. With an inclination of about 45 degrees the dipole effect can be minimized to $\left(\mathrm{U}-\mathrm{U}_{\mathrm{AV}}\right) / \mathrm{U}_{\mathrm{AV}}=0.4 \%$ (see table 2), A dipole of $2 \%$ or less is insignificant [2], it is below the experimental tuning tolerances of RFQs.

\begin{tabular}{|c|c|}
\hline Dipole / $\%$ & $\alpha /$ Degrees \\
\hline 1.41 & 0 \\
\hline 1.17 & 10.2 \\
\hline 1.04 & 19.9 \\
\hline 0.89 & 28.5 \\
\hline 0.76 & 35.9 \\
\hline 0.60 & 43.9 \\
\hline 0.45 & 50.3 \\
\hline
\end{tabular}

Table 2: Dipole-effects versus shape of stems.

The dipole-effects can be reduced by longer stems too, at a lower frequency. Table 3 shows the decrease of dipoles as function of the length of the stems (rectangular cross-section).

\begin{tabular}{|c|c|}
\hline Length of Stems / mm & Dipole / \% \\
\hline 600 & 1.27 \\
\hline 622 & 1.22 \\
\hline 644 & 1.18 \\
\hline 666 & 1.14 \\
\hline 688 & 1.10 \\
\hline 710 & 1.06 \\
\hline
\end{tabular}

Table 3: Dipole-effects versus length of stems. 

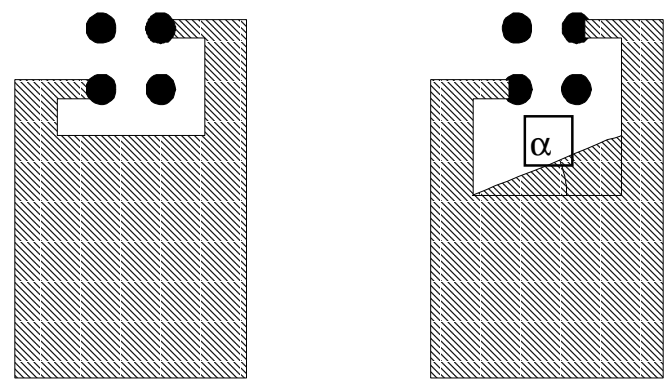

Fig. 1: Shape of four rod RFQ stems.

\section{Alternative structures}

Alternative structures are the spiral loaded RFQ, which has been successfully tested $(27 \mathrm{MHz})$ and reached full beam current recently at GSI [3]. There, this structure was felt to be not stable enough, so that the structure with the linear straight stems discussed above was favoured at first.

Another structure was proposed, which can be characterized as an Interdigital-H-shaped resonator loaded with Four-Rod electrodes and supports [4]. To study this resonator, a model scaled to about $100 \mathrm{MHz}$ has been built.

This model showed dipole effects of $8 \%$, what scales to $2 \%$ at a frequency of $36 \mathrm{MHz}$. No distinctly advantage compared to the stems-type RFQ can be seen, whereas other technical difficulties may appear.

MAFIA-calculations may show different results for dipoles because of different paths of integration. Integration paths, shown in fig. 2, give voltage values, which are in good agreement with experiments. Direct integration on the beam axis typically has too small values, smaller than the errors of the MAFIA code.
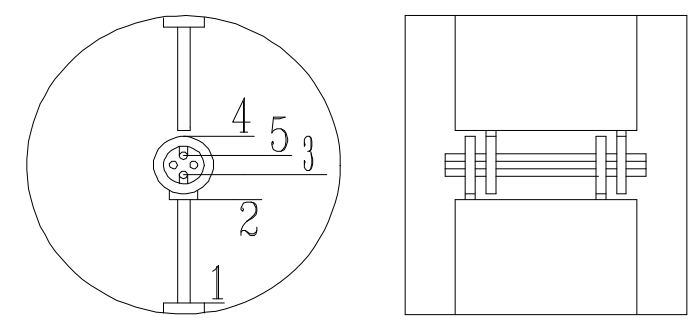

Fig. 2: Interdigital-H-structure.

\section{COOLING CONSIDERATIONS}

Due to the maximum duty factor of about $3 \%$ the rfpower distribution has been checked. Less than $20 \%$ of the losses appear on the electrodes. Nevertheless this is critical, because of the mechanical precision required. In a stem-RFQ with distances of $400 \mathrm{~mm}$ the electrodes will have to be cooled with water like at the HLI-RFQ, which operates at
$25 \%$ duty factor [5]. For omitting the cooling, the stems must be arranged with smaller distances, e. g. $250 \mathrm{~mm}$.

Experiments with the $27 \mathrm{MHz}$ Spiral loaded RFQ have shown that water cooled electrodes are not necessary anyhow, because the conduction of heat by the electrode carriers is sufficient [3].

For a higher frequency (e. g. $200 \mathrm{MHz}$ at ESS- or ATWprojects) the average power is higher ( $5 \%$ duty factor up to c. w., $5-100 \mathrm{~kW} / \mathrm{m})$. A high duty factor prototype with a frequency of $200 \mathrm{MHz}$ is being built at IAP Frankfurt for experiments on cooling and stability.

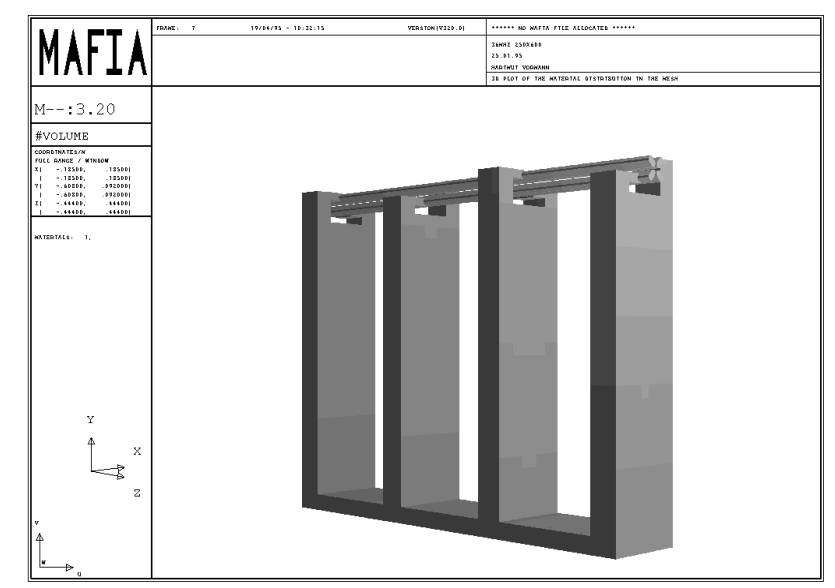

Fig. 3: MAFIA-Plot of the stems-prototype.

\section{A. Distribution of losses}

Measurements with four-rod RFQs have been confirmed by MAFIA-calculations: The RFQ losses are distributed as follows: $17 \%$ on the electrodes, $9 \%$ on the blocks where the electrodes are fixed to the stems (electrode carriers), $58 \%$ on the stems, $7 \%$ on the base plate and only $9 \%$ on the walls of the cavity, which is one of the big advantages for high power operation.

\section{BEAM DYNAMICS}

The low input energy of $2.2 \mathrm{keV} / \mathrm{N}$ allows a relatively short RFQ with a sufficient number of cells to achieve a convenient beam formation for good beam quality. The RFQ has to be designed for a short bunch width and a small energy spread at the output and a minimum transverse emittance growth.

In figs. 4 and 5 the results of beam dynamics calculations are shown. The calculations have been done with the program PARMTEQ for an input beam with transverse waterbag distribution and no initial energy spread. In fig. 4 the emittances behind the RFQ are shown for a normalized input emittance of $0.3 \pi \mathrm{mm}$ mrad. The transmission is $90 \%$, the emittance growth only $5.5 \%$. 
RFQ FOR U 4, F=36.1MHZ, $U=125 \mathrm{KV}$

NCELL $=356$, NPOINT $=1810$, NTOTAL $=2000, \pi i n=15 \mathrm{~mA}$
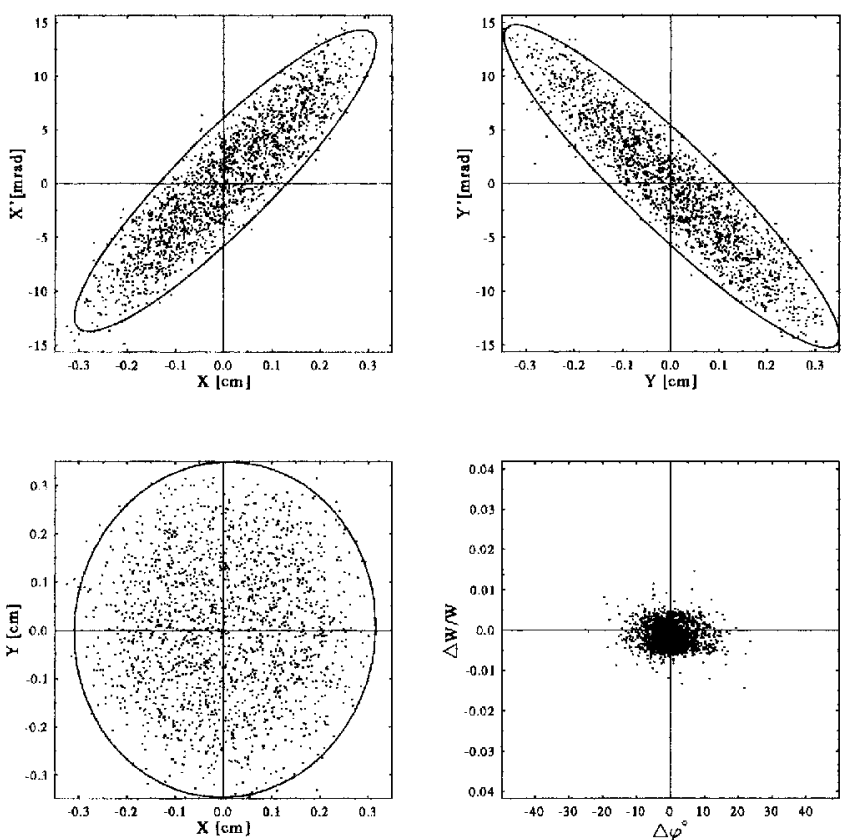

Fig. 4: RFQ output emittances, with space charge (15 mA).

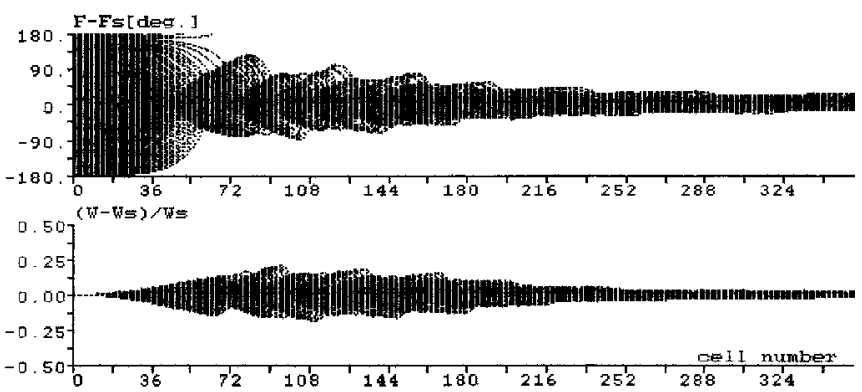

Fig. 5: Beam transmission along the RFQ.

\section{A. RF-Power Requirements}

An expected Rp-value of $500 \mathrm{k} \Omega \mathrm{m}$ enables the operator of an RFQ with a length of 9 meter $(=55 \mathrm{k} \Omega)$ to reach an electrode voltage of $125 \mathrm{kV}$ with an rf-power of $280 \mathrm{~kW}$.

Table 4 shows a comparison of different RFQ-resonators. The stems have rectangular cross-section with different width and length, except number 5 which has stems with a circular cross-section of $120 \mathrm{~mm}$, and the IH-structure with $15 \mathrm{~mm}$ distance of the supports of the electrodes, according to the GSI design. [4]

The low power model has a simple geometry: The stems with a cross-section of $200 \mathrm{~mm} \times 10 \mathrm{~mm}$ have a distance of $400 \mathrm{~mm}$ and a height of $400 \mathrm{~mm}$. The electrodes are optional cylindric with a radius of $5 \mathrm{~mm}$ or vane-like with a shape of $10 \mathrm{~mm} \times 20 \mathrm{~mm}$, each $1240 \mathrm{~mm}$ long, the aperture is $10 \mathrm{~mm}$. The resonator was only screwed, so bad contacts reduce the shuntimpedance. Table 5 shows the comparison between calculation and measurements.

\begin{tabular}{|c|c|c|c|}
\hline Stems & $\begin{array}{c}\text { Frequency / } \\
\mathrm{MHz}\end{array}$ & $\begin{array}{c}\text { Quality- } \\
\text { Factor }\end{array}$ & $\begin{array}{c}\text { Shunt- } \\
\text { impedance }\end{array}$ \\
\hline $400 \times 400$ & 30.7 & 10400 & 770 \\
\hline $250 \times 600$ & 36.9 & 13000 & 790 \\
\hline $250 \times 700$ & 34.6 & 12900 & 830 \\
\hline $250 \times$ rad 60 & 33.5 & 11500 & 840 \\
\hline IH-Structure & 37.1 & 8000 & 360 \\
\hline
\end{tabular}

Table 4: Properties of different RFQ-resonators.

\begin{tabular}{|c|c|c|}
\hline & Measurement & MAFIA \\
\hline Frequency / MHz & 34.3 & 32.2 \\
\hline Quality-Factor & 2900 & 10500 \\
\hline Shuntimp./ k $\Omega \mathrm{m}$ & 270 & 770 \\
\hline
\end{tabular}

Table 5: Comparison of calculated and measured properties.

\section{CONCLUSIONS}

Four-rod type RFQs for low frequencies have high efficiency, good mechanical stability, good operational characteristics and suffer no dipole mode problems, which has been shown in calculations, prototypes and operating structures. Spiral shaped and straight stems supports give compact resonators. GSI will go on working with the IHdriven four-rod RFQ, for which prototyping on a number of design issues is the next stage.

\section{REFERENCES}

[1]A. Schempp et al, " A Light Ion Four-Rod RFQ Injector“, Proc. IEEE PAC 1987, p. 267,

[2] J. Klabunde et al, "Beam Dynamics Simulation in a FourRod RFQ“, Proc. Linac 1994, p. 710,

[3] U. Bessler et al, "Status of the beam experiments with the $27 \mathrm{MHz}-$ Spiral-RFQ at GSI", to be published,

[4] U. Ratzinger, GSI Note, HSP 27 104.STR, 1994,

[5] J. Klabunde et al., "Upgrade of the HLI RFQ Accelerator", Proc. Linac 1994, p. 704.. 